\title{
Extreme Dark Matter Tests with Extreme Mass Ratio Inspirals
}

\author{
Otto A. Hannuksela, ${ }^{1, *}$ Kenny C. Y. ${ }^{2 g},{ }^{2, \dagger}$ and Tjonnie G. F. $\mathrm{Li}^{1, \ddagger}$ \\ ${ }^{1}$ Department of Physics, The Chinese University of Hong Kong, Sha Tin, N.T., Hong Kong \\ ${ }^{2}$ Department of Particle Physics and Astrophysics, Weizmann Institute of Science, Rehovot, Israel
}

(Dated: June 27, 2019)

\begin{abstract}
Future space-based laser interferometry experiments such as LISA are expected to detect $\mathcal{O}(100-1000)$ stellarmass compact objects (e.g., black holes, neutron stars) falling into massive black holes in the centers of galaxies, the so-called extreme-mass-ratio inspirals (EMRIs). If dark matter forms a "spike" due to the growth of the massive black hole, it will induce a gravitational drag on the inspiraling object, changing its orbit and gravitationalwave signal. We show that detection of even a single dark matter spike from the EMRIs will severely constrain several popular dark matter candidates, such as ultralight bosons, keV fermions, MeV-TeV self-annihilating dark matter, and sub-solar mass primordial black holes, as these candidates would flatten the spikes through various mechanisms. Future space gravitational wave experiments could thus have a significant impact on the particle identification of dark matter.
\end{abstract}

\section{INTRODUCTION}

Astrophysical and cosmological observations from vastly different scales have established the existence of non-baryonic substance — dark matter (DM) - that makes up around $85 \%$ of all known matter [1, 2]. Significant effort has gone into identifying DM, whose discovery will be a crucial breakthrough in fundamental physics and understandings of the Universe.

Many probes of DM utilize regions of high DM densities, such as the Galactic halo and dwarf spheroidals (dSphs). One attractive target for such searches is the DM spikes, dense concentrations of DM surrounding massive black holes $(\mathrm{BH})$ in centers of galaxies [3-5], formed as the BHs grow adiabatically. If DM self-annihilates, the spikes would significantly boost the annihilation rate. This has led to searches of bright isolated gamma-ray sources in the sky as well as constraints in DM annihilation cross sections [6-17]. However, the abundance of the BHs and the DM spike properties (notably the density slope) are uncertain $[6,18-20]$. The sub-parsec regions of the BHs can only be probed in a few selected systems [21-23]. Furthermore, there is no guarantee that DM can self-annihilate. This dual uncertainty makes it difficult to constrain either the DM spikes or DM particle properties robustly.

Thankfully, this picture could change dramatically soon. With the advent of gravitational wave (GW) astronomy, future space laser interferometry experiments, such as LISA [24], Taiji [25], and TianQin [26] can detect hundreds to thousands of stellar-mass compact objects (BHs, neutrinos stars, and white dwarfs) falling into intermediate or supermassive BHs [27]. These events are called extreme mass ratio inspirals (EMRIs). GWs from EMRIs can probe the properties of the surrounding DM spikes [28-32], or similar concentrations [32]. By measuring the DM spike profile using purely gravitational interactions, it is possible to reliably detect the spike and then simultaneously constrain the properties of DM.

Previously, it was demonstrated that EMRI measurements could be used to infer ultralight boson properties [32]. The potential to test DM properties were also briefly noted in Refs. [2, 28, 29]. However, a clear picture of the implications of EMRI detection to various DM models is absent.

In this Letter, we show that even with a single GW detection of DM spike, one can place strong constraints on the properties of several popular DM models. These include ultralight bosons, keV fermions, self-annihilating DM, and primordial BHs. We provide the principal arguments and orderof-magnitude, but robust, determination of the constraints.

\section{DM SPIKE FORMATION AROUND BHS}

We consider the massive BHs that sit at the centers of DM halos. The density profile near the $\mathrm{BH}$ is approximated by

$$
\rho(r) \simeq \rho_{0}\left(r / r_{0}\right)^{-\gamma} .
$$

When the BH grows adiabatically, the surrounding DM density also changes according to the changes in the gravitational pull and forms a spike $[3,4,33]$ :

$$
\rho_{\mathrm{sp}}(r)=\rho_{R}\left(1-8 M_{\mathrm{BH}} / r\right)^{3}\left(r / R_{\mathrm{sp}}\right)^{-\alpha},
$$

where $\alpha=(9-2 \gamma) /(4-\gamma)$, which yields $2.25<\alpha<2.5$ for $0<\gamma<2$ (here and below we will use units of $G=c=\hbar=1$ ). The factors $\rho_{R}$ and $R_{\mathrm{sp}}$ depend on $\gamma$ and the $\mathrm{BH}$ mass $M_{\mathrm{BH}}$ through the $M-\sigma$ relation ([33], and see Appendix). $\gamma=1$ corresponds to the NFW profile, and we consider $\gamma=2$ to be the optimistic case.

We note that depending on the merger history and the stellar environment of the BHs, the DM spikes could be disrupted and end up with shallower final slopes [6, 18, 34-36]. However, the extent of these effects is still being debated [12, 13], and it has been suggested that spikes around lighter BHs are less affected by mergers $[37,38]$. We emphasize that the EMRI GW detection itself does not depend on the formation scenarios or properties of DM (e.g., annihilation), and thus provides a model-independent test for the spikes.

Figure 1 shows the peak spike density $\rho_{\text {peak }} \approx$ $\rho_{\mathrm{sp}}\left(20 M_{\mathrm{BH}}\right)$ for $M_{\mathrm{BH}} \in\left[10^{3}, 10^{6}\right] M_{\odot}$. For $\gamma=1, \rho_{\text {peak }}$ can reach up to $10^{23} \mathrm{GeV} / \mathrm{cm}^{2}$. For the optimistic $\gamma=2$ scenario, $\rho_{\text {peak }}$ can reach up to $10^{26} \mathrm{GeV} / \mathrm{cm}^{2}$. If detected, 
the DM spikes will be the most compact DM structures ever known.

\section{DM SPIKE DETECTION WITH EMRI}

We now turn to the detection prospects of DM spikes with GWs from EMRI events.

Detecting the DM Spikes We model GWs from extreme mass-ratio binary systems interacting with DM spikes (following Refs. [28, 29]), and recover the spike parameters in LISA setting. As the compact objects fall into the BHs, they lose energy and change orbits due to dynamical friction. The properties of the DM spike are thus encoded in the emitted GWs.

Our set-up is as follows: For each BH mass, $M_{\mathrm{BH}}$, we set a constant signal-to-noise ratio of SNR $=30$ (the usual detection threshold for EMRIs [39]). Dark matter effects are modeled at the lowest post-Newtonian (PN) order for $\rho_{\mathrm{dm}}(r)=$ $\rho_{\text {peak }}\left(r / 20 M_{\mathrm{BH}}\right)^{-\alpha}$ profile (as in Ref. [29]). Other binary interactions are at 2.5 PN [40]. We set the mass ratio of the binary $q=\mu / M_{\mathrm{BH}}=10^{-4}$, and we assume LISA sensitivity with angle-averaged antenna pattern functions [41]. We then recover the parameters of the injected waveforms, including $\rho_{\text {peak }}$ and the density slope $\alpha$, by the Fisher Information Matrix (FIM) method $[40]^{1}$. We assume 5 years of orbital time and the last orbital cycle at $r=20 M_{\mathrm{BH}}$.

Figure 1 shows the expected $\rho_{\text {peak }}$ recovery uncertainty for the above setup. The peak density can be recovered at a high accuracy $\left(\Delta \log _{10} \rho_{\text {peak }} \ll 1\right)$ for large ranges of $\rho_{\text {peak }}$ and $M_{\mathrm{BH}}$. However, we emphasize (and show below) even order-of-magnitude estimates $\left(\Delta \log _{10} \rho_{\text {peak }} \lesssim 3\right)$ will be enough for us to place stringent bounds on the DM models. Specifically, we find that $\Delta \log _{10} \rho_{\text {peak }} \lesssim 3$ at BH masses $M_{\mathrm{BH}} \in\left[10^{3}, 10^{4.5}\right] \mathrm{M}_{\odot}$ and $M_{\mathrm{BH}} \in\left[10^{3}, 10^{6}\right]$ for $\gamma=1$ and $\gamma=2$, respectively. We consider the cases where $\Delta \log _{10} \rho_{\text {peak }}>3$, as "unmeasurable." At all the considered values, the spike leaves a noticeable orbital shift on the compact object's trajectory and therefore is measurable in gravitational wave emission. Below $10^{3} M_{\odot}$, the detector sensitivity deteriorate rapidly [42].

We account for degeneracies between the $2.5 \mathrm{PN}$ binary parameters and the DM spike parameters by the FIM approach. More accurate waveforms introduce higher order effects. In principle, these corrections could be degenerate with the gravitational drag induced by the spike. However, we expect the degeneracies to be small: the spike introduces a slow cumulative phase shift due to gravitational drag, which is quite distinct from the higher order binary effects such as spin precession.

\footnotetext{
${ }^{1}$ Specifically, we recover the parameters $\vec{\theta} \in$ $\left\{A, \phi_{c}, t_{c}, \log \mathcal{M}_{c}, \log \eta, \beta, \sigma, \rho_{\text {peak }}, \alpha\right\}$, where $\beta, \quad \sigma \quad$ represent spin-orbit and spin-spin contributions to the phasing [40]
}

We note that our results do not strongly depend on the final spike index, but is most sensitive to $\rho_{\text {peak }}$. For simplicity, we fix the SNR and $q$. A more realistic, larger SNR would lower the measurable line (in Fig. 1), e.g., by a factor of a few for $\mathrm{SNR}=100$. The detectability also improves by a factor of a few if we choose $q=10^{-3}$, and vice versa for $q=10^{-5}$. However, we note that our waveform approximation likely breaks down at smaller values of $q\left(q \ll 10^{-4}\right)$. We leave the more accurate and detailed exploration of the parameter space with for future work.

Finally, we note that in our default DM spike model, the final density index is always $\alpha>2.25$. If the astrophysical spike disruption effects are important, the slope could be flattened, perhaps ending with a shallow case $\alpha \simeq 1.5$ or an intermediate case $\alpha \simeq 1.8$ [12]. Even in the shallow case, the spike can still be detectable at $\left\{M_{\mathrm{BH}}=10^{3} M_{\odot}, \gamma=\right.$ $2\}$; while in the intermediate case, the spike is detectable at $\left\{M_{\mathrm{BH}}=10^{3} M_{\odot}, \gamma \simeq 1.6\right\}$ or $\left\{M_{\mathrm{BH}} \leq 10^{4} M_{\odot}, \gamma=2\right\}$. The EMRI GW probe is thus sensitive to a broad range of spike parameters.

EMRI detection rates Studies of the expected EMRI rate with self-consistent $\mathrm{BH}$ formation and evolution models suggest $\mathcal{O}(100-1000)$ EMRI events throughout the lifetime of LISA [27]. Importantly, these models suggest a mildly negative BH mass function, thus favors events with lighter BHs. $\mathrm{DM}$ spikes around lighter $\mathrm{BHs}$ are denser and thus easier to detect (Fig. 1). Also, light BHs are less likely to suffer from major mergers and are thus more likely to retain the DM spikes $[28,29,37,38]$.

\section{DARK MATTER TESTS WITH EMRI}

If DM consists of ultralight bosons, light fermions, selfannihilating particles, or primordial $\mathrm{BHs}$ (PBHs), the DM spike density would be affected. Measuring the DM spike with EMRI could test these scenarios simultaneously.

Ultralight bosonic DM: Heisenberg's uncertainty principle causes the light bosons (with mass $\mu_{s}$ ) to dilute the DM spikes and set a theoretical upper bound on their density. Such theoretical upper bound on density allows us to constrain the light boson mass by spike observations.

Consider an initial scalar field $\psi_{i}$ surrounding the center of a galaxy without a black hole. Since the boson mass is light, it forms a BEC (i.e., it is in its ground state; $\psi_{i}=\psi_{i}^{\text {ground }}$ for some initial Hamiltonian) [43-48].

A black hole then grows adiabatically in the center, evolving the surrounding scalar field. The growth takes place on much larger timescale than the scalar field $\mathrm{cycle}^{2}$, thus adiabatic theorem [53-55] applies: The final state of the scalar field $\psi_{f}$, after growth, will be in the ground state of the final

\footnotetext{
${ }^{2}$ The scalar field oscillation time-scale is $\tau \sim \mu_{s}^{-1} \lesssim 100 \mathrm{yr} \ll 10^{6} \mathrm{yrs}$ when $\mu_{s} \gtrsim 10^{25} \mathrm{eV}$ [49-52]
} 


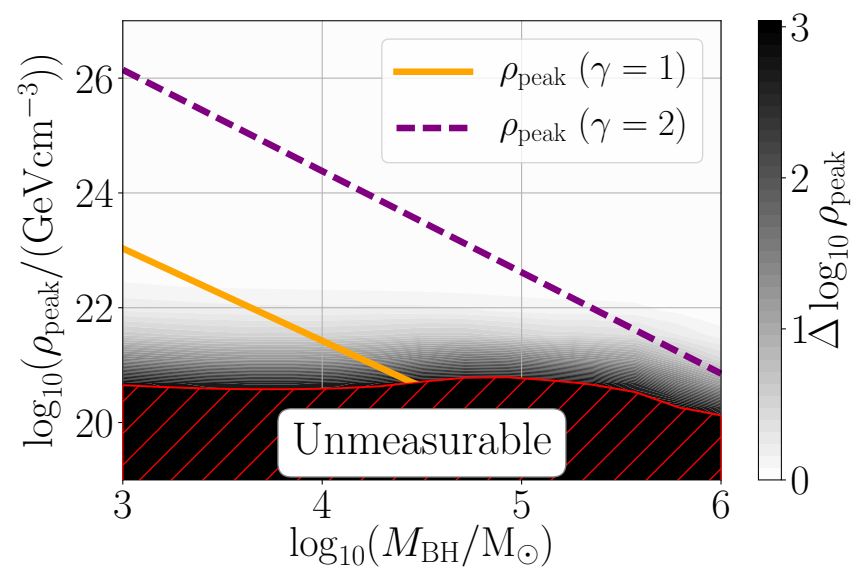

FIG. 1. Uncertainty in the peak DM spike density $\Delta \log _{10} \rho_{\text {peak }}$ from EMRI GW measurement around a massive $\mathrm{BH}$ (grey scale) as a function of $\rho_{\text {peak }}$ and $\mathrm{BH}$ mass $M_{\mathrm{BH}}$. We set the mass ratio $q=10^{-4}$ and signal-to-noise ratio $\mathrm{SNR}=30$. In several cases, we can recover the peak density at high accuracy $\left(\Delta \log _{10} \rho_{\max } \ll\right.$ 1). However, we emphasize that even order-of-magnitude estimates $\left(\Delta \log _{10} \rho_{\max } \lesssim 3\right.$ ) will be enough to place stringent constraints on the DM models. $\Delta \log _{10} \rho_{\max } \lesssim 3$ is satisfied in the range $M_{\mathrm{BH}} \in\left[10^{3}, 10^{4.5}\right] \mathrm{M}_{\odot}$ and $M_{\mathrm{BH}} \in\left[10^{3}, 10^{6}\right] \mathrm{M}_{\odot}$ for $\gamma=1$ and $\gamma=2$ (thick solid and dashed lines, respectively). Beyond that, we consider the event "unmeasurable." We apply Gaussian filter for data visualization purposes.

Hamiltonian, which is approximately the black hole Hamiltonian near the center [44].

Thus, the final state after growth is the ground state of a black hole/boson system, which has an exact solution in the very light boson mass limit $\left(M_{\mathrm{BH}} \mu_{s} \ll 1\right)$ [49]. The density of the ground state is $[49,56]$ :

$\rho_{s}\left(\mu_{s}, M_{\mathrm{BH}}, r, \theta, M_{s}\right) \simeq \frac{M_{s}}{64 \pi \mu_{s}} M_{\mathrm{BH}}^{5} \mu_{s}^{11} r^{2} e^{-M_{\mathrm{BH}} \mu_{s}^{2} r} \sin ^{2} \theta$,

where $r$ is the radius, $M_{s}=\int \rho r^{2} d r d \Omega$ is the cloud mass, and the density has been expanded in leading order of $r^{-1}$. Note that we assume complex scalar fields, but real fields share similar predictions [50, 51, 57, 58].

The cloud mass must be smaller than the mass inside the influence radius $\left(M_{s} \lesssim M_{s}^{\max } \sim 2 M_{\mathrm{BH}}\right.$ [62]). This translates to constraints on the maximal observed density $\rho_{\text {obs }} \lesssim$ $\rho_{\max }=\rho_{s}\left(\mu, M_{\mathrm{BH}}, 2 /\left(M_{\mathrm{BH}} \mu_{s}^{2}\right), \pi / 2, M_{s}^{\max }\right)$, which yields

$\mu_{s} \gtrsim 10^{-17} \mathrm{eV}\left(\frac{\rho_{\mathrm{obs}}}{10^{20} \mathrm{GeV} / \mathrm{cm}^{3}} \frac{2 M_{\mathrm{BH}}}{M_{s}^{\max }}\right)^{1 / 6}\left(\frac{10^{6} \mathrm{M}_{\odot}}{M_{\mathrm{BH}}}\right)^{2 / 3}$.

Consequently, we could disfavour, for example, the fuzzy/wave DM candidate in the $\mu_{s} \in\left[10^{-23}, 10^{-21}\right] \mathrm{eV}$ range $[47,48]$, proposed as a solution to many cosmological problems (Fig. 2, panel a).

We note that if stars or compact objects of large mass are present near the $\mathrm{BH}$ and in sufficient abundance, it is possible that the boson cloud ground state (Eq. 3) could mix with higher order states or collapse back to the $\mathrm{BH}[56,63]$. In this case, the boson cloud will no longer reside in the ground state, but either does not exist or resides a higher state. However, this is not an issue: Higher modes are spread across an even larger volume and therefore predict smaller densities [49], making our estimate conservative.

Note also that when the boson mass is larger ${ }^{3}$, superradiance can create bosonic clouds [51,52]. These clouds may allow one to verify the existence of the bosons by this same EMRI measurement as shown in Ref. [32] (we show the target region in Figure 2, panel a, green area, for completeness).

Fermionic DM: If DM is made of fermions, such as sterile neutrinos, they occupy a finite phase space volume due to the exclusion principle. Thus, a measurement of the mass density can be used to derive robust limits on fermionic DM mass.

Consider a system of a degenerate fermionic DM, the Fermi velocity is

$$
v_{F}=\left(\frac{6 \pi^{2} \hbar^{3} \rho}{m_{\mathrm{DM}}^{4} g}\right)^{1 / 3} .
$$

For the density spike to be stable, the Fermi velocity must be less than the escape velocity of the BH plus DM spike system

$$
v_{F} \leq v_{e s c} \equiv \sqrt{\frac{2 G\left(M_{\mathrm{BH}}+M_{\chi}\right)}{R}} \simeq \sqrt{\frac{2 G M_{\mathrm{BH}}}{R}} .
$$

This translates to a lower bound on the fermionic DM mass, given an observation of density $\rho_{\mathrm{obs}}$,

$$
m_{\mathrm{DM}} \gtrsim 30 \mathrm{keV}\left(\frac{\rho_{\mathrm{obs}}}{10^{20} \mathrm{GeV} / \mathrm{cm}^{3}} \frac{2}{g}\right)^{1 / 4}\left(\frac{R}{20 M_{\mathrm{BH}}}\right)^{3 / 8} .
$$

Thus, a detection of the DM spike will significantly improve existing fermioninc DM constraint [60] by more than 2 orders of magnitude. While our result only depends on the measured density, we express the constraint in terms of $M_{\mathrm{BH}}$ using our reference DM spike model for consistency, as shown in Fig. 2 panel a. This result is robust, and does not depend on the initial phase-space density distribution [64, 65]. It will also close the $\nu$ MSM sterile neutrino DM window $[66,67]$ without relying on X-ray searches [68-74].

Self-annihilating DM: Thermally produced weakly interacting massive particles is a popular generic DM candidate, and predicts a definite annihilation cross section $(\sim$ $10^{-26} \mathrm{~cm}^{3} \mathrm{~s}^{-1}$ for s-wave, $2 \rightarrow 2$ ) that is required to produced the observed DM abundance [75]. The self-annihilation would also rapidly smooth out the DM spike into "annihilation plateaus" [4]. Thus, observation of the DM spike places a limit on the annihilation cross section $\sigma v$.

We approximate the annihilation plateau as a flat core, $\rho_{\text {core }}=m_{\chi} /\left(\sigma v t_{\mathrm{BH}}\right)[3]$ (see also [17, 36, 76]). Taking conservatively the age of the $\mathrm{BH}$ to be $t_{\mathrm{BH}} \gtrsim 10^{6}$ years (much

\footnotetext{
${ }^{3}$ In the LISA BH range $M_{\mathrm{BH}} \in\left[10^{3}, 10^{6}\right] \mathrm{M}_{\odot}$ would have such "matching" bosons in the $\mu_{s} \in\left[10^{-17}, 10^{-14}\right] \mathrm{eV}$ range.
} 

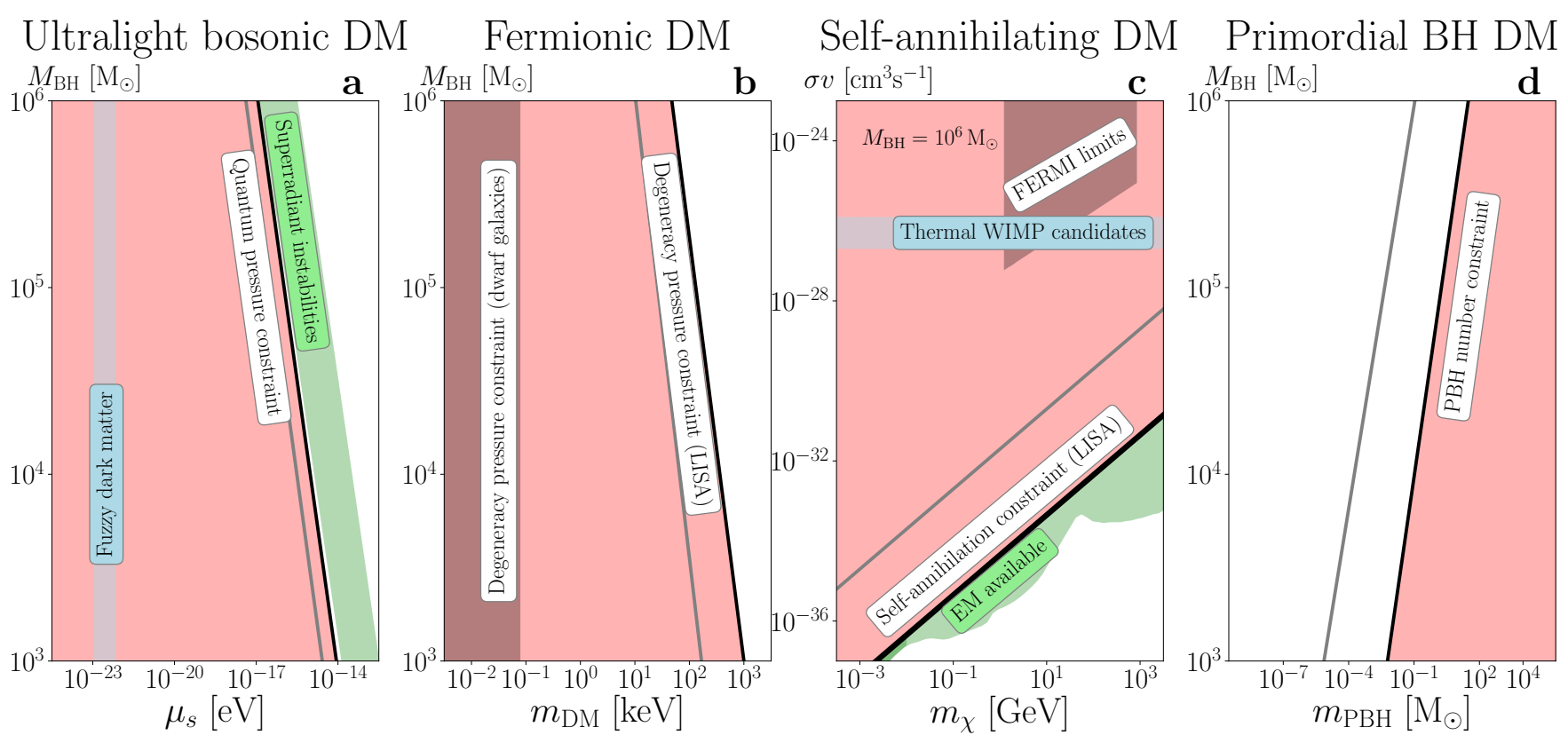

FIG. 2. New constraints (red shaded regions) on DM models if a DM spike is detected with an EMRI. For ultralight bosons (panel a), fermionic DM (panel b), and PBH DM (panel d), we exclude a region of the DM particle/PBH mass. The constraints depend on the mass of the detected central black hole, $M_{\mathrm{BH}}$ (the DM spike profile is uniquely predicted for given $M_{\mathrm{BH}}$ using the $M-\sigma$ relation). For self-annihilating DM (panel c), the constraint is on the cross section-DM mass plane, assuming $M_{\mathrm{BH}}=10^{6} \mathrm{M}_{\odot}$. If ultralight bosons exist in the $m_{\mathrm{DM}} \in\left[10^{-17}, 10^{-14}\right]$ $\mathrm{eV}$ range, they could be identified through superradiant-induced clouds (see Ref. [32]; panel a, green region). If the EMRI event is sufficiently nearby $(\simeq 90 \mathrm{Mpc})$, as expected of the closest EMRIs [59], electromagnetic counterparts from DM annihilation may be possible in some optimistic cases (panel c, green region). Previous lower limits (gray regions) on fermionic DM and upper limits on DM annihilation cross section are from Refs. [60, 61]. For all panels, the thick solid lines and thin gray lines correspond to $\gamma=2$ and $\gamma=1$ initial DM halo slopes, respectively. See text and Appendix for details.

less than the mean age of galaxies or stars, $\sim 10^{10} \mathrm{yr}$ ), an EMRI DM spike measurement sets a upper limit on the total annihilation cross section,

$$
\begin{aligned}
\sigma v & \lesssim 3.17 \times 10^{-32} \mathrm{~cm}^{3} \mathrm{~s}^{-1}\left(\frac{m_{\chi}}{100 \mathrm{GeV}}\right) \\
& \times\left(\frac{10^{20} \mathrm{GeV} / \mathrm{cm}^{3}}{\rho_{\mathrm{obs}}}\right)\left(\frac{10^{6} \mathrm{yr}}{t_{\mathrm{BH}}}\right) .
\end{aligned}
$$

Thus, any EMRI DM spike measurement will be in strong tension with the simplest thermal relic DM hypothesis (Fig. 2, panel c), currently an open window between $20 \mathrm{GeV}<m_{\chi}<$ $100 \mathrm{TeV}$ [61]. We emphasize this is the total cross section, thus includes the difficult-to-probe neutrino channels.

For other cases (p-wave annihilation, non-thermal models, and others), the cross-section could be significantly lower [13]. In such scenarios, the EMRI event could have a persistent electromagnetic counterpart due to DM annihilation. We find that in the optimistic scenario, where $\gamma=$ 2 , the $\mathrm{BH}$ is heavy $M_{\mathrm{BH}} \sim 10^{6} \mathrm{M}_{\odot}$ and nearby $D \sim$ $90 \mathrm{Mpc}$, the electromagnetic counterpart is detectable with eASTROGAM/Fermi/CTA (see the Appendix for details), as shown in Fig. 2. However, within such a small volume, the expected number of EMRI events is only of order one [59]. Thus, to see the electromagnetic counterpart, the fraction of halos hosting spikes must be high, the DM spike must be relatively young, and the event must be nearby.
Primordial black hole DM We consider the case that PBHs dominates cosmic DM density (as long as the non-PBH component of DM cannot mimic a spike as described above.) If a DM spike is measured with EMRI, there must be at least one PBH $(N \geq 1)$ in the probed volume $\left(8 M_{\mathrm{BH}}<r<\right.$ $\left.300 M_{\mathrm{BH}}\right)$. The mass of the of the $\mathrm{PBH}, m_{\mathrm{PBH}}$, must then satisfy

$$
N m_{\mathrm{PBH}} \leq \int_{8 M_{\mathrm{BH}}}^{300 M_{\mathrm{BH}}} \rho_{\mathrm{obs}}(r) d^{3} r .
$$

Consequently, the PBH mass range could be constrained to $m_{\mathrm{PBH}} \lesssim 10^{-7} \mathrm{M}_{\odot}(\gamma=1$, Fig. 2, panel d). This simple PBH number argument offers an independent constraint on the PBHs, complementary to various existing considerations (e.g., Refs. [77-82]).

We note that the above $N=1$ constraint is exceptionally conservative. In the case of a spike detection, for the PBH DM to mimic the dynamical friction effect, the spike must have a large amount of PBHs $N \gg 1$, thus leading to more stringent constraints. We leave the exploration of this effect for the future.

In principle, one could also combine these spike measurements with ground-based detectors that observe PBH mergers within the spikes. Unfortunately, the fraction of mergers within a single halo is $N_{\mathrm{sp}} \lesssim 10^{-2} \mathrm{yr}^{-1}$ (Ref. [33]]), and thus 
constraining PBHs by aid of ground-based detectors will be difficult. We note that PBHs themselves also act as EMRIs, which could offer another channel for their detections [83].

\section{DISCUSSION AND CONCLUSION}

We have shown that, in the near future, EMRI GW measurement with space interferometry experiments could place strong constraints on DM models across the particle landscape. The EMRI GW emission will provide modelindependent (purely gravitational) tests for DM spikes, which is a prediction of cold collisionless DM models. If such spikes are detected, they will be the most compact DM structures ever known.

These compact structures are incompatible with several popular DM models, such as ultra-light bosons, keV fermions, self-annihilating DM, and PBHs. Note that in the DM parameter space we consider here, the DM spike will always be flattened due to the intrinsic DM particle properties. On the other hand, as discussed earlier, astrophysical effects may also partially flatten the spike. A large number of expected EMRIs ( 100-1000) is thus extremely advantageous; it allows EMRIs to probe $\mathrm{BH}$ systems under various astrophysical conditions and with variable merger histories, reducing the chance of non-detection of the spike due to astrophysical effects.

If no spikes are detected, then, unfortunately, no conclusions can be drawn immediately. One possibility is that astrophysical processes (stellar heating, mergers, etc [6, 18, 20, 35]) had destroyed the spikes. The processes must then be common, robust, and applicable to different BHs masses and galaxy properties. Follow-up and detailed astronomical observations of the EMRI events (e.g., [84]) are then necessary to identify the astrophysical mechanism. Indeed, galaxies with large EMRI rates may tend to have stellar cusps, which could potentially negatively bias their likelihood of hosting DM spikes [27]. The properties of DM might also cause the smoothing of the spike. In these cases, the GW observation itself may already contain smoking-gun signatures of the particle DM (e.g.,[32]). Otherwise, independent probes are needed to pinpoint the particle effect (e.g., gamma-ray searches or others [22, 23]).

Here we provide the principal methodologies for DM searches by EMRIs. To fully realize the search potential, realistic waveforms $[27,85,86]$ that can capture the effect of DM are required. These waveforms would need to be suitable for the extreme gravity and mass ratio that we consider here, and should also be generic in their ability to distinguish DM distributions. The form of the spike distribution can in principle be quite generic, and therefore more flexible waveforms, specialized towards EMRIs, should be developed before they can be applied to realistic LISA data analysis.

Despite decades of searches, the identity of DM is still elusive. If DM spikes are detected, they could provide much-needed sensitivity to revolutionize the landscape of DM searches.

\section{ACKNOWLEDGMENTS}

We thank Emanuele Berti, Richard Brito, Kaze W. K. Wong for their work on a very closely related project, Yifan Wang for discussion on primordial black holes and Ming-Chung Chu and Po Kin Leung for their supervision on a project on dark matter spike modeling. We also thank John F. Beacom for his helpful comments, Richard Brito for his advice on ultralight bosons, and Kaze W. K. Wong for his comments on data-analysis. O.A.H. is supported by the Hong Kong Ph.D. Fellowship Scheme (HKPFS) issued by the Research Grants Council (RGC) of Hong Kong. K.C.Y.N. is supported by a Croucher Fellowship and a Benoziyo Fellowship. T.G.F.L. was partially supported by grants from the Research Grants Council of the Hong Kong (Project No. CUHK14310816, CUHK24304317 and CUHK14306218), The Croucher Foundation in Hong Kong, and the Research Committee of the Chinese University of Hong Kong.

\section{Appendix}

\section{Relating the dark matter spike profile to the host black hole}

When a BH surrounded by $\rho_{i}(r) \simeq \rho_{0}\left(r_{0} / r\right)^{\gamma}$ DM profile grows adiabatically, it gravitationally concentrates the surrounding matter around it to a cuspy profile usually referred to as a "DM spike" [3]. The DM density spike can be modelled generally as [33]

$$
\rho_{\mathrm{sp}}(r)=\rho_{R}\left(1-\frac{8 M_{\mathrm{BH}}}{r}\right)^{3}\left(\frac{r}{R_{\mathrm{sp}}}\right)^{-\alpha}
$$

where $\rho_{R}=\rho_{0}\left(R_{\mathrm{sp}} / r_{0}\right)^{-\gamma}, \quad R_{\mathrm{sp}}=$ $a_{\gamma} r_{0}\left(M_{\mathrm{BH}} /\left(\rho_{0} r_{0}^{3}\right)\right)^{1 /(3-\gamma)}, \quad a_{\gamma}$ is a numerical fit with $a_{1} \simeq 0.1$ and $a_{2} \simeq 0.02$ [3] and $\gamma_{\mathrm{sp}}=(9-2 \gamma) /(4-\gamma)$. The parameters $\rho_{0}$ and $r_{0}$ can be related to the initial density profile outside the spike, taken to be the NFW profile, via the $\mathrm{BH}$ mass through the $M-\sigma$ relationship, the one-dimensional halo velocity dispersion and the virial mass relations [33]

$$
\begin{aligned}
\log _{10}\left(M_{\mathrm{BH}} / \mathrm{M}_{\odot}\right) & =a+b \log _{10}\left(\sigma / 200 \mathrm{kms}^{-1}\right) \\
\sigma^{2} & =\frac{4 \pi G \rho_{0} r_{0}^{2} g\left(c_{m}\right)}{c_{m}} \\
M_{\mathrm{vir}} & =4 \pi \rho_{0} r_{0}^{3} g\left(c\left(M_{\mathrm{vir}}\right)\right) \\
M_{\mathrm{vir}} & =\frac{4}{3} \pi R_{\mathrm{vir}}^{3} \Delta \rho_{c}
\end{aligned}
$$

where $a=8.12$ and $b=4.24$ are empirically determined parameters, $\sigma$ is the one-dimensional halo velocity dispersion of the galaxy, $g(x)=\log (1-x)-x /(1+x)$, which is obtain by the mass the mass integral of the NFW profile (Eq. 13), $c_{m}=2.16, \Delta \simeq 200$, and $\rho_{c}$ is the critical density. The 
concentration ( $\left.c=R_{v i r} / r_{0}\right)$ relation is [87]

$$
\begin{aligned}
c\left(M_{\mathrm{vir}}\right) & =c_{5} \log ^{5}\left(\frac{h_{0} M_{\mathrm{vir}}}{\mathrm{M}_{\odot}}\right)+c_{4} \log ^{4}\left(\frac{h_{0} M_{\mathrm{vir}}}{\mathrm{M}_{\odot}}\right) \\
& +c_{3} \log ^{3}\left(\frac{h_{0} M_{\mathrm{vir}}}{\mathrm{M}_{\odot}}\right)+c_{2} \log ^{2}\left(\frac{h_{0} M_{\mathrm{vir}}}{\mathrm{M}_{\odot}}\right) \\
& +c_{1} \log \left(\frac{h_{0} M_{\mathrm{vir}}}{\mathrm{M}_{\odot}}\right)+c_{0},
\end{aligned}
$$

where $h_{0}=0.67, c_{0}=37.5153, c_{1}=-1.5093, c_{2}=$ $1.636 \times 10^{-2}, c_{3}=3.66 \times 10^{-4}, c_{4}=-2.89237 \times 10^{-5}$ and $c_{5}=5.32 \times 10^{-7}$. Using Eqs. 11-15, we use the $M-\sigma$ relation to connect $M_{\mathrm{BH}}$ and $M_{\mathrm{vir}}$

$$
\sigma_{v}^{2}\left(M_{\mathrm{BH}}\right)=\frac{4 \pi}{3} G \Delta \rho_{c} \frac{g\left(c_{\mathrm{m}}\right) c}{g(c) c_{\mathrm{m}}}\left(\frac{3 M_{\mathrm{vir}}}{4 \pi \Delta \rho_{c}}\right)^{2 / 3} .
$$

We can then solve for $M_{\mathrm{vir}}$ numerically for each $M_{\mathrm{BH}}$, and subsequently obtain $\rho_{R}$ and $R_{\mathrm{sp}}$.

We note that the inner part of the density profile (roughly outside the spike) is not well constrained [88]. We, therefore, choose two reference values for our profile; $\gamma=1$, which corresponds to the NFW profile. In some cases [89], the density slope could be steeper. We thus consider $\gamma=2$ to be the most optimistic scenario.

\section{Electromagnetic Counterparts from DM annihilations}

For DM annihilation in the DM spike, the differential gamma-ray flux is

$$
\frac{d F}{d E}=\frac{\sigma v}{2 m_{\chi}^{2}} \frac{\int \rho_{\mathrm{sp}}^{2} d V}{4 \pi D^{2}} \frac{d N}{d E}
$$

where $\sigma v$ is the annihilation cross section, $m_{\chi}$ is the DM mass, $D$ is the distance to the source, and $d N / d E$ is the gamma-ray spectrum per annihilation. For simplicity, we assume $100 \% \chi \chi \rightarrow \gamma \gamma$ below $10 \mathrm{GeV}$ and $\chi \chi \rightarrow \tau \tau$ above. For the $\gamma \gamma$ channel, we assume delta function spectrum convolved with $10 \%$ energy resolution, a typical detector resolution for gamma-ray detectors. For the $\tau \tau$ channel, we obtain the spectrum from PPPC4DMID [90].

For detecting the gamma-ray flux, we consider the joint differential sensitivity in $1 \mathrm{MeV}-10 \mathrm{TeV}$ from 1 year of eASTROGAM [91], 10 years of Fermi [92], and 50 hours of CTA [93].

We obtain the sensitivity (green band, Fig. 2, panel c) requiring the DM flux to touch the detector sensitivity described above. This is conservative, as in practice sharp spectra from DM annihilation typically can improve the sensitivity by a factor of a few, after taking into account the smooth astrophysical and detector backgrounds.

\section{Parameter inference of the dark matter distribution}

Our GW waveform strain is of the form

$$
h(f)=\frac{\sqrt{3}}{2} A f^{-7 / 6} e^{i\left(\psi(f)+\psi_{\mathrm{DM}}(f)\right)},
$$

where $f$ is the GW frequency, $A=\mathcal{M}_{c}^{5 / 6} /\left[\left(30^{1 / 2} \pi^{2 / 3}\right) d_{L}\right]$ is the amplitude with $\mathcal{M}_{c}$ being the chirp mass. The phase of the GW $\psi(f)$ is approximated up to 2.5 post-Newtonian order as in [40].

$$
\begin{aligned}
\psi(f)= & 2 \pi f t_{c}-\phi_{c} \\
& +\frac{3}{128} X^{-5 / 3}\left(1+\left(\frac{55 \eta}{9}+\frac{3715}{756}\right) \eta^{-2 / 5} X^{2 / 3}\right. \\
& +\left(\frac{3085 \eta^{2}}{72}+\frac{27145 \eta}{504}+\frac{15293365}{508032}\right) \eta^{-4 / 5} X^{4 / 5} \\
& \left.+4 \beta \eta^{-3 / 5} X-16 \pi \eta^{-3 / 5} X-10 \eta^{-4 / 5} \sigma X^{4 / 3}\right),
\end{aligned}
$$

where $t_{c}$ and $\phi_{c}$ are the time and phase of coalescence, $X=$ $\pi \mathcal{M}_{c} f, \eta$ is the symmetric mass ratio and $(\beta, \sigma)$ are the binary spin parameters. In all our calculations, we assume angleaveraged LISA antenna pattern functions.

We construct the phase shift introduced by a DM spike $\psi_{\mathrm{DM}}(f)$ similarly to Ref. [29]. We choose the DM spike of the form of

$$
\rho_{\mathrm{dm}}(r)=\rho_{\text {peak }}\left(\frac{r}{20 M_{\mathrm{BH}}}\right)^{-\alpha},
$$

where $\rho_{\text {peak }}, \alpha$ are the spike parameters. The spike introduces a gravitational pull and a dynamical friction onto the binary orbit $[29,94]$

$$
\frac{d E_{\mathrm{orbit}}}{d t}+\frac{d E_{\mathrm{gw}}}{d t}+\frac{d E_{\mathrm{DF}}}{d t}=0
$$

where

$$
\begin{aligned}
\frac{d E_{\mathrm{orbit}}}{d t} & =\frac{d}{d t}\left(\frac{1}{2} \mu v^{2}+\mu \Phi(r)\right), \\
\frac{d E_{\mathrm{gw}}}{d t} & =\frac{32 \mu^{2} r^{4} \omega^{6}}{5} \\
\frac{d E_{\mathrm{DF}}}{d t} & =\frac{4 \pi \mu^{2} \log \Lambda \rho_{\mathrm{dm}}(r)}{v(r)}
\end{aligned}
$$

with $v$ and $\omega$ being the velocity and orbital frequency of the smaller compact object, $\log \Lambda \sim 3+$ $\log \left[\left(10^{-4} / q\right)\left(20 M_{\mathrm{BH}} / r\right)\right]$ is the Coulomb logarithm [95] which depends weakly on the mass ratio $q$ and orbital radius $r$ [95], $\mu$ the reduced mass of the system (which we take to be approximately the component mass), and $\Phi(r)$ is the Newtonian potential of the spike and the black hole.

The most dominant effect that the collisionless dark matter introduces is dynamical friction; the accretion onto the black hole [31] (which we ignore) and gravitational pull are both 
sub-dominant [30, 31]. We assume a constant value of the Coulomb logarithm throughout the inspiral, as in [29], computed at $r=20 M_{\mathrm{BH}}$ and given mass ratio $q$.

Dynamical friction stems from the gravitational pull by high-density "tails" that form behind the compact object in its wake. We note that ultralight bosons could, in principle, inhibit the formation of these tails due to quantum effects, similarly as they inhibit the formation of high-density peaks within galaxies [47]. However, the precise framework to model the gravitational effects by light bosons (or more generally scalar fields) is still being worked out [94, 96-103]. If the tails and thus dynamical friction are indeed inhibited, then a measurement of the spike would imply even stronger constraints on the ultralight bosons.

We obtain the rate of change of orbital radius $r^{\prime}(t)$ from the energy evolution equation. Inverting the orbital frequency yields $r(f)$ [29], which can be translated to the total GW phase shift using the stationary phase approximation [104].

The mapping between the orbital radius and the GW frequency may be solved by inverting the following relation for $r$

$$
\omega(f)=\pi f=\sqrt{\frac{\Phi^{\prime}(r)}{\mu r}},
$$

and expanding to first order in matter effects (Ref. [29]).

We then estimate the parameter recovery using standard Fisher Information Matrix (FIM) approach, which applies in the high signal-to-noise ratio limit (as implemented in $[40,105])$, and assume LISA power spectral density [41]. The recovered parameters are $\vec{\theta} \in$ $\left\{A, \phi_{c}, t_{c}, \log \mathcal{M}_{c}, \log \eta, \beta, \sigma, \rho_{0}, \alpha\right\}$.

Let us note a common issue with the FIM approach: If the FIM is high-dimensional, the FIM inversion can become unstable for specific sets of parameters [40, 106]. In Fig. 1, we exclude these unstable sets of parameters in our analysis and replace them by values interpolated from the nearest stable points.

\section{Adiabatic theorem}

Consider wave function of two non-degenerate states in the non-relativistic limit, such that we can separate the wave function of the bosonic cloud in two components (similarly to $[56,63])$

$$
|\psi(t)\rangle=c_{n}(t) e^{i \theta_{n}(t)}\left|\psi_{n}(t)\right\rangle+c_{m}(t) e^{i \theta_{m}(t)}\left|\psi_{m}(t)\right\rangle,
$$

such that $\left|c_{n}(t)\right|^{2}+\left|c_{m}(t)\right|^{2}=1$ and $c_{n}(0)=1$ and $c_{m}(0)=$ 0 . The exponential phase is introduced for convenience and satisfies $\theta_{n}(t)=-\int_{0}^{t} \omega_{n}\left(t^{\prime}\right) d t^{\prime}$.

Inserting this into the Schrödinger equation, we find

$$
\sum_{j=n, m} e^{i \theta_{j}(t)}\left[\dot{c}_{j}(t)\left|\psi_{j}(t)\right\rangle+c_{j}(t) \partial_{t}\left|\psi_{j}(t)\right\rangle\right]=0 .
$$

Taking inner product with respect to $\langle m|$, we get

$$
\dot{c}_{m}(t)=-\sum_{j=n, m} c_{j}(t) e^{i\left[\theta_{j}(t)-\theta_{m}(t)\right]}\left\langle\psi_{m}(t)\right|\left[\partial_{t}\left|\psi_{j}(t)\right\rangle\right] .
$$

Inserting the following generic result for Schrödinger equation

$$
\left\langle\psi_{m}(t)|\dot{H}| \psi_{n}(t)\right\rangle=\left[\omega_{n}(t)-\omega_{m}(t)\right]\left\langle\psi_{m}(t)\left|\partial_{t}\right| \psi_{j}(t)\right\rangle,
$$

we get

$$
\begin{aligned}
\dot{c}_{m}(t)= & -c_{m}(t)\left\langle\psi_{m}(t)\left|\partial_{t}\right| \psi_{m}(t)\right\rangle \\
& -c_{n}(t) e^{i\left(\theta_{n}(t)-\theta_{m}(t)\right)} \frac{\left\langle\psi_{m}(t)|\dot{H}| \psi_{n}(t)\right\rangle}{\omega_{n}-\omega_{m}},
\end{aligned}
$$

where the second term can be neglected whenever [e.g. 53]

$$
\frac{\left\langle\psi_{m}(t)|\dot{H}| \psi_{n}(t)\right\rangle}{\omega_{n}-\omega_{m}} \equiv \frac{1}{\tau} \ll\left\langle\psi_{m}(t)\right|\left[\partial\left|\psi_{m}(t)\right\rangle\right] \sim \omega_{m} .
$$

I.e., the Hamiltonian changes over a much longer period of time than the oscillation frequency of the system, which for given $(n l m)$ state is $[56,63]$

$$
\omega_{n \ell m} \simeq \mu\left(1-\frac{\alpha^{2}}{2 n^{2}}+\delta \omega_{n \ell m}\right)
$$

where $\delta \omega_{n \ell m}$ denote higher-order corrections, that (up to fifth-order in $\alpha$ ) are given by [56, 63]

$$
\begin{aligned}
\delta \omega_{n \ell m} \simeq & \left(-\frac{\alpha^{4}}{8 n^{4}}+\frac{(2 \ell-3 n+1) \alpha^{4}}{n^{4}(\ell+1 / 2)}\right. \\
& \left.+\frac{2 \tilde{a} m \alpha^{5}}{n^{3} \ell(\ell+1 / 2)(\ell+1)}\right),
\end{aligned}
$$

where $\tilde{a}=a / M_{\mathrm{BH}}$ is the dimensionless spin. The ground state in this notation is $n=1, l=0, m=0$ state. To first order, the oscillation time-scale is

$$
\omega_{n \ell m}^{-1} \sim 2 \mathrm{yr}\left(\frac{\mu}{10^{-23} \mathrm{eV}}\right),
$$

which is much smaller than the black hole growth time-scale $\left(\sim 10^{6} \mathrm{yr}\right)$. The adiabatic growth assumption is only violated at $\mu \ll 10^{-25} \mathrm{eV}$ mass range.

As a cautionary note, the ground state is to a large degree degenerate with different $m$ modes: $m=-1,0,1$. This could potentially cause mode mixing of the stable ground mode with different (unstable) $m$ modes. However, we can safely neglect the mixing, as these states are both similarly diluted as the ground state. Hence, our estimate (Eq. 3) is conservative.

\footnotetext{
* hannuksela@phy.cuhk.edu.hk; orcid.org/0000-0002-3887-7137

† chun-yu.ng@weizmann.ac.il; orcid.org/0000-0001-8016-2170

‡ tgfli@ cuhk.edu.hk; orcid.org/0000-0003-4297-7365
} 
[1] Gianfranco Bertone, Dan Hooper, and Joseph Silk. Particle dark matter: Evidence, candidates and constraints. Physics Reports, 405(5):279-390, 2005.

[2] Gianfranco Bertone and Tim MP Tait. A new era in the search for dark matter. Nature, 562(7725):51, 2018.

[3] Paolo Gondolo and Joseph Silk. Dark Matter Annihilation at the Galactic Center. Physical Review Letters, 83(9):17191722, August 1999.

[4] Laleh Sadeghian, Francesc Ferrer, and Clifford M Will. Darkmatter distributions around massive black holes: A general relativistic analysis. Physical Review D, 88(6):063522, 2013.

[5] Francesc Ferrer, Augusto Medeiros da Rosa, and Clifford M. Will. Dark matter spikes in the vicinity of Kerr black holes. Phys. Rev., D96(8):083014, 2017.

[6] David Merritt, Milos Milosavljević, Licia Verde, and Raul Jimenez. Dark Matter Spikes and Annihilation Radiation from the Galactic Center. Physical Review Letters, 88(19):191301, April 2002.

[7] F. Aharonian et al. Search for Gamma-rays from Dark Matter annihilations around Intermediate Mass Black Holes with the H.E.S.S. experiment. Phys. Rev., D78:072008, 2008.

[8] Gianfranco Bertone, Mattia Fornasa, Marco Taoso, and Andrew R. Zentner. Dark Matter Annihilation around Intermediate Mass Black Holes: an update. New J. Phys., 11:105016, 2009.

[9] Torsten Bringmann, Julien Lavalle, and Pierre Salati. Intermediate Mass Black Holes and Nearby Dark Matter Point Sources: A Myth-Buster. Phys. Rev. Lett., 103:161301, 2009.

[10] Brian C. Lacki and John F. Beacom. Primordial Black Holes as Dark Matter: Almost All or Almost Nothing. Astrophys. J., 720:L67-L71, 2010.

[11] Máximo Bañados, Babiker Hassanain, Joseph Silk, and Stephen M. West. Emergent flux from particle collisions near a Kerr black hole. Physical Review D, 83(2):023004, January 2011.

[12] Brian D. Fields, Stuart L. Shapiro, and Jessie Shelton. Galactic Center Gamma-Ray Excess from Dark Matter Annihilation: Is There A Black Hole Spike? Phys. Rev. Lett., 113:151302, 2014.

[13] Jessie Shelton, Stuart L. Shapiro, and Brian D. Fields. Black hole window into $p$-wave dark matter annihilation. Phys. Rev. Lett., 115(23):231302, 2015.

[14] Thomas Lacroix, Céline Bœhm, and Joseph Silk. Ruling out thermal dark matter with a black hole induced spiky profile in the M87 galaxy. Physical Review D, 92(4):043510, August 2015.

[15] Mark Wanders, Gianfranco Bertone, Marta Volonteri, and Christoph Weniger. No WIMP mini-spikes in dwarf spheroidal galaxies. Journal of Cosmology and Astroparticle Physics, 2015(04):004, 2015.

[16] Pearl Sandick, Kuver Sinha, and Takahiro Yamamoto. Black Holes, Dark Matter Spikes, and Constraints on Simplified Models with t-Channel Mediators. arXiv:1701.00067 [astroph, physics:hep-ph], December 2016. arXiv: 1701.00067.

[17] Stuart L. Shapiro and Jessie Shelton. Weak annihilation cusp inside the dark matter spike about a black hole. Physical Review D, 93(12), June 2016. arXiv: 1606.01248.

[18] Piero Ullio, HongSheng Zhao, and Marc Kamionkowski. A Dark-Matter Spike at the Galactic Center? Physical Review $D, 64(4)$, July 2001. arXiv: astro-ph/0101481.

[19] David Merritt. Evolution of the dark matter distribution at the galactic center. Physical review letters, 92(20):201304, 2004.

[20] Gianfranco Bertone and David Merritt. Time-Dependent Models for Dark Matter at the Galactic Center. Physical Re- view $D, 72(10)$, November 2005. arXiv: astro-ph/0501555.

[21] Thomas Lacroix, Mansour Karami, Avery E. Broderick, Joseph Silk, and Celine Boehm. A unique probe of dark matter in the core of M87 with the Event Horizon Telescope. arXiv:1611.01961 [astro-ph, physics:hep-ph], November 2016. arXiv: 1611.01961.

[22] Nitsan Bar, Kfir Blum, Thomas Lacroix, and Paolo Panci. Looking for ultralight dark matter near supermassive black holes. 2019.

[23] Hooman Davoudiasl and Peter B Denton. Ultra Light Boson Dark Matter and Event Horizon Telescope Observations of M87*. 2019.

[24] Pau Amaro-Seoane et al. Laser Interferometer Space Antenna. arXiv preprint arXiv:1702.00786, 2017.

[25] Zong-Kuan Guo, Rong-Gen Cai, and Yuan-Zhong Zhang. Taiji Program: Gravitational-Wave Sources. 2018.

[26] Jun Luo et al. TianQin: a space-borne gravitational wave detector. Class. Quant. Grav., 33(3):035010, 2016.

[27] Stanislav Babak, Jonathan Gair, Alberto Sesana, Enrico Barausse, Carlos F. Sopuerta, Christopher P. L. Berry, Emanuele Berti, Pau Amaro-Seoane, Antoine Petiteau, and Antoine Klein. Science with the space-based interferometer LISA. V: Extreme mass-ratio inspirals. Phys. Rev., D95(10):103012, 2017.

[28] Kazunari Eda, Yousuke Itoh, Sachiko Kuroyanagi, and Joseph Silk. New probe of dark-matter properties: gravitational waves from an intermediate-mass black hole embedded in a dark-matter minispike. Physical review letters, 110(22):221101, 2013.

[29] Kazunari Eda, Yousuke Itoh, Sachiko Kuroyanagi, and Joseph Silk. Gravitational waves as a probe of dark matter minispikes. Physical Review D, 91(4):044045, 2015.

[30] Enrico Barausse, Vitor Cardoso, and Paolo Pani. Environmental effects for gravitational-wave astrophysics. In Journal of Physics: Conference Series, volume 610, page 012044. IOP Publishing, 2015.

[31] Xiao-Jun Yue and Wen-Biao Han. Gravitational waves with dark matter minispikes: The combined effect. Physical Review $D, 97(6): 064003,2018$.

[32] Otto A Hannuksela, Kaze WK Wong, Richard Brito, Emanuele Berti, and Tjonnie GF Li. Probing the existence of ultralight bosons with a single gravitational-wave measurement. Nature Astronomy, page 1, 2019.

[33] Hiroya Nishikawa, Ely D Kovetz, Marc Kamionkowski, and Joseph Silk. Primordial-black-hole mergers in dark-matter spikes. arXiv preprint arXiv:1708.08449, 2017.

[34] Taro Nakano and Junichiro Makino. On the cusp around central black holes in luminous elliptical galaxies. The Astrophysical Journal Letters, 525(2):L77, 1999.

[35] Gianfranco Bertone and David Merritt. Dark matter dynamics and indirect detection. Modern Physics Letters A, 20(14):1021-1036, 2005.

[36] Eugene Vasiliev and Maxim Zelnikov. Dark matter dynamics in the galactic center. Physical Review D, 78(8):083506, 2008.

[37] HongSheng Zhao and Joseph Silk. Dark minihalos with intermediate mass black holes. Physical review letters, 95(1):011301, 2005.

[38] Gianfranco Bertone, Andrew R Zentner, and Joseph Silk. New signature of dark matter annihilations: Gamma rays from intermediate-mass black holes. Physical Review D, 72(10):103517, 2005.

[39] Jonathan R Gair, Leor Barack, Teviet Creighton, Curt Cutler, Shane L Larson, E Sterl Phinney, and Michele Vallisneri. Event rate estimates for lisa extreme mass ratio capture 
sources. Classical and Quantum Gravity, 21(20):S1595, 2004.

[40] Emanuele Berti, Alessandra Buonanno, and Clifford M. Will. Estimating spinning binary parameters and testing alternative theories of gravity with LISA. Phys. Rev., D71:084025, 2005.

[41] Neil Cornish and Travis Robson. The construction and use of LISA sensitivity curves. 2018.

[42] Jonathan R Gair, Stanislav Babak, Alberto Sesana, Pau Amaro-Seoane, Enrico Barausse, Christopher PL Berry, Emanuele Berti, and Carlos Sopuerta. Prospects for observing extreme-mass-ratio inspirals with lisa. In Journal of Physics: Conference Series, volume 840, page 012021. IOP Publishing, 2017.

[43] Wayne Hu, Rennan Barkana, and Andrei Gruzinov. Fuzzy cold dark matter: the wave properties of ultralight particles. Physical Review Letters, 85(6):1158, 2000.

[44] L Arturo Urena-Lopez and Andrew R Liddle. Supermassive black holes in scalar field galaxy halos. Physical Review D, 66(8):083005, 2002.

[45] Andrew P Lundgren, Mihai Bondarescu, Ruxandra Bondarescu, and Jayashree Balakrishna. Lukewarm dark matter: Bose condensation of ultralight particles. The Astrophysical Journal Letters, 715(1):L35, 2010.

[46] Alejandro Cruz-Osorio, F Siddhartha Guzmán, and Fabio D Lora-Clavijo. Scalar field dark matter: behavior around black holes. Journal of Cosmology and Astroparticle Physics, 2011(06):029, 2011.

[47] Hsi-Yu Schive, Tzihong Chiueh, and Tom Broadhurst. Cosmic structure as the quantum interference of a coherent dark wave. Nature Physics, 10(7):496-499, 2014.

[48] Jiajun Zhang, Yue-Lin Sming Tsai, Jui-Lin Kuo, Kingman Cheung, and Ming-Chung Chu. Ultralight axion dark matter and its impact on dark halo structure in n-body simulations. The Astrophysical Journal, 853(1):51, 2018.

[49] Steven Detweiler. Klein-gordon equation and rotating black holes. Physical Review D, 22(10):2323, 1980.

[50] Asimina Arvanitaki, Savas Dimopoulos, Sergei Dubovsky, Nemanja Kaloper, and John March-Russell. String axiverse. Physical Review D, 81(12):123530, 2010.

[51] Richard Brito, Vitor Cardoso, and Paolo Pani. Superradiance. Lect. Notes Phys, 906(1):18, 2015.

[52] Richard Brito, Vitor Cardoso, and Paolo Pani. Black holes as particle detectors: evolution of superradiant instabilities. Classical and Quantum Gravity, 32(13):134001, 2015.

[53] Jun John Sakurai. Advanced quantum mechanics. Pearson Education India, 1967.

[54] G Nenciu. On the adiabatic theorem of quantum mechanics. Journal of Physics A: Mathematical and General, 13(2):L15, 1980.

[55] Ali Mostafazadeh. Adiabatic geometrical phase for scalar fields in a curved spacetime. arXiv preprint hep-th/9608051, 1996.

[56] Daniel Baumann, Horng Sheng Chia, and Rafael A Porto. Probing ultralight bosons with binary black holes. Physical Review D, 99(4):044001, 2019.

[57] Sam R. Dolan. Instability of the massive Klein-Gordon field on the Kerr spacetime. Phys. Rev., D76:084001, 2007.

[58] Asimina Arvanitaki, Masha Baryakhtar, and Xinlu Huang. Discovering the qcd axion with black holes and gravitational waves. Physical Review D, 91(8):084011, 2015.

[59] Jonathan R Gair. Probing black holes at low redshift using lisa emri observations. Classical and Quantum Gravity, 26(9):094034, 2009.

[60] Chiara Di Paolo, Fabrizio Nesti, and Francesco L. Villante. Phase space mass bound for fermionic dark matter from dwarf spheroidal galaxies. Mon. Not. Roy. Astron. Soc., 475(4):5385-5397, 2018.

[61] Rebecca K Leane, Tracy R Slatyer, John F Beacom, and Kenny CY Ng. Gev-scale thermal wimps: Not even slightly dead. arXiv preprint arXiv:1805.10305, 2018.

[62] David Merritt. Single and binary black holes and their influence on nuclear structure. In Carnegie Observatories Centennial Symposium. 1. Coevolution of Black Holes and Galaxies Pasadena, California, October 20-25, 2002, 2003.

[63] Emanuele Berti, Richard Brito, Caio FB Macedo, Guilherme Raposo, and João Luís Rosa. Ultralight boson cloud depletion in binary systems. Physical Review D, 99(10):104039, 2019.

[64] S. Tremaine and J. E. Gunn. Dynamical Role of Light Neutral Leptons in Cosmology. Phys. Rev. Lett., 42:407-410, 1979.

[65] Shunsaku Horiuchi, Philip J. Humphrey, Jose Onorbe, Kevork N. Abazajian, Manoj Kaplinghat, and Shea GarrisonKimmel. Sterile neutrino dark matter bounds from galaxies of the Local Group. Phys. Rev., D89(2):025017, 2014.

[66] Laurent Canetti, Marco Drewes, and Mikhail Shaposhnikov. Sterile Neutrinos as the Origin of Dark and Baryonic Matter. Phys. Rev. Lett., 110(6):061801, 2013.

[67] Laurent Canetti, Marco Drewes, Tibor Frossard, and Mikhail Shaposhnikov. Dark Matter, Baryogenesis and Neutrino Oscillations from Right Handed Neutrinos. Phys. Rev., D87:093006, 2013.

[68] Esra Bulbul, Maxim Markevitch, Adam Foster, Randall K. Smith, Michael Loewenstein, and Scott W. Randall. Detection of An Unidentified Emission Line in the Stacked X-ray spectrum of Galaxy Clusters. Astrophys. J., 789:13, 2014.

[69] Alexey Boyarsky, Oleg Ruchayskiy, Dmytro Iakubovskyi, and Jeroen Franse. Unidentified Line in X-Ray Spectra of the Andromeda Galaxy and Perseus Galaxy Cluster. Phys. Rev. Lett., 113:251301, 2014.

[70] Kenny C. Y. Ng, Shunsaku Horiuchi, Jennifer M. Gaskins, Miles Smith, and Robert Preece. Improved Limits on Sterile Neutrino Dark Matter using Full-Sky Fermi Gamma-Ray Burst Monitor Data. Phys. Rev., D92(4):043503, 2015.

[71] Eric G. Speckhard, Kenny C. Y. Ng, John F. Beacom, and Ranjan Laha. Dark Matter Velocity Spectroscopy. Phys. Rev. Lett., 116(3):031301, 2016.

[72] Devon Powell, Ranjan Laha, Kenny C. Y. Ng, and Tom Abel. Doppler effect on indirect detection of dark matter using dark matter only simulations. Phys. Rev., D95(6):063012, 2017.

[73] Kerstin Perez, Kenny C. Y. Ng, John F. Beacom, Cora Hersh, Shunsaku Horiuchi, and Roman Krivonos. Almost closing the $\nu$ MSM sterile neutrino dark matter window with NuSTAR. Phys. Rev., D95(12):123002, 2017.

[74] Kenny C. Y. Ng, Brandon M. Roach, Kerstin Perez, John F. Beacom, Shunsaku Horiuchi, Roman Krivonos, and Daniel R. Wik. New Constraints on Sterile Neutrino Dark Matter from NuST AR M31 Observations. Phys. Rev., D99:083005, 2019.

[75] Gary Steigman, Basudeb Dasgupta, and John F. Beacom. Precise Relic WIMP Abundance and its Impact on Searches for Dark Matter Annihilation. Phys. Rev., D86:023506, 2012.

[76] Eugene Vasiliev. Dark matter annihilation near a black hole: Plateau versus weak cusp. Physical Review D, 76(10):103532, 2007.

[77] Fabio Capela, Maxim Pshirkov, and Peter Tinyakov. Constraints on primordial black holes as dark matter candidates from capture by neutron stars. Physical Review D, 87(12):123524, 2013.

[78] Daniel Aloni, Kfir Blum, and Raphael Flauger. Cosmic microwave background constraints on primordial black hole dark matter. JCAP, 1705(05):017, 2017. 
[79] Mathieu Boudaud and Marco Cirelli. Voyager $1 e^{ \pm}$Further Constrain Primordial Black Holes as Dark Matter. Phys. Rev. Lett., 122(4):041104, 2019.

[80] William DeRocco and Peter W. Graham. Constraining primordial black hole abundance with the Galactic $511 \mathrm{keV}$ line. 2019.

[81] Ranjan Laha. Primordial black holes as dark matter candidate are severely constrained by the Galactic Center $511 \mathrm{keV}$ gamma-ray line. 2019.

[82] Guillermo Ballesteros, Javier Coronado-Blázquez, and Daniele Gaggero. X-ray and gamma-ray limits on the primordial black hole abundance from Hawking radiation. 2019.

[83] Florian Kuhnel, Andrew Matas, Glenn D Starkman, and Katherine Freese. Waves from the centre: Probing pbh and other macroscopic dark matter with lisa. arXiv preprint arXiv:1811.06387, 2018.

[84] Kazunori Akiyama et al. First M87 Event Horizon Telescope Results. I. The Shadow of the Supermassive Black Hole. Astrophys. J., 875(1):L1, 2019.

[85] Stanislav Babak, Hua Fang, Jonathan R. Gair, Kostas Glampedakis, and Scott A. Hughes. 'Kludge' gravitational waveforms for a test-body orbiting a Kerr black hole. Phys. Rev., D75:024005, 2007. [Erratum: Phys. Rev.D77,04990(2008)].

[86] Alvin J. K. Chua, Christopher J. Moore, and Jonathan R. Gair. Augmented kludge waveforms for detecting extreme-massratio inspirals. Phys. Rev., D96(4):044005, 2017.

[87] Miguel A Sánchez-Conde and Francisco Prada. The flattening of the concentration-mass relation towards low halo masses and its implications for the annihilation signal boost. Monthly Notices of the Royal Astronomical Society, 442(3):2271-2277, 2014.

[88] Miguel Pato, Fabio Iocco, and Gianfranco Bertone. Dynamical constraints on the dark matter distribution in the milky way. Journal of Cosmology and Astroparticle Physics, 2015(12):001, 2015.

[89] Oleg Y Gnedin, Andrey V Kravtsov, Anatoly A Klypin, and Daisuke Nagai. Response of dark matter halos to condensation of baryons: cosmological simulations and improved adiabatic contraction model. The Astrophysical Journal, 616(1):16, 2004.

[90] Marco Cirelli, Gennaro Corcella, Andi Hektor, Gert Hutsi, Mario Kadastik, Paolo Panci, Martti Raidal, Filippo Sala, and Alessandro Strumia. PPPC 4 DM ID: A Poor Particle Physicist Cookbook for Dark Matter Indirect Detection. JCAP, 1103:051, 2011. [Erratum: JCAP1210,E01(2012)].
[91] M. Tavani et al. Science with e-ASTROGAM: A space mission for MeV-GeV gamma-ray astrophysics. JHEAp, 19:1106, 2018.

[92] http://www.slac.stanford.edu/exp/glast/groups/canda /lat_Performance.htm.

[93] www.cta-observatory.org.

[94] Caio FB Macedo, Paolo Pani, Vitor Cardoso, and Luís CB Crispino. Into the lair: gravitational-wave signatures of dark matter. The Astrophysical Journal, 774(1):48, 2013.

[95] James Binney and Scott Tremaine. Galactic dynamics. Princeton university press, 2011.

[96] Michael Kesden, Jonathan Gair, and Marc Kamionkowski. Gravitational-wave signature of an inspiral into a supermassive horizonless object. Physical Review D, 71(4):044015, 2005.

[97] Ricardo Becerril, Tonatiuh Matos, and Luis Ureña-López. Geodesics around oscillatons. General Relativity and Gravitation, 38(4):633-641, 2006.

[98] Valeria Diemer, Keno Eilers, Betti Hartmann, Isabell Schaffer, and Catalin Toma. Geodesic motion in the space-time of a noncompact boson star. Physical Review D, 88(4):044025, 2013.

[99] Caio FB Macedo, Paolo Pani, Vitor Cardoso, and Luís CB Crispino. Astrophysical signatures of boson stars: quasinormal modes and inspiral resonances. Physical Review D, 88(6):064046, 2013.

[100] Yves Brihaye, Valeria Diemer, and Betti Hartmann. Charged q-balls and boson stars and dynamics of charged test particles. Physical Review D, 89(8):084048, 2014.

[101] Philippe Grandclement, Claire Somé, and Eric Gourgoulhon. Models of rotating boson stars and geodesics around them: new type of orbits. Physical Review D, 90(2):024068, 2014.

[102] Mateja Bošković, Francisco Duque, Miguel C Ferreira, Filipe S Miguel, and Vitor Cardoso. Motion in time-periodic backgrounds with applications to ultralight dark matter halos at galactic centers. Physical Review D, 98(2):024037, 2018.

[103] Miguel C Ferreira. How do scalar-field dark matter halos react to orbiting bodies? Physical Review D, 99(10):103008, 2019.

[104] Michele Maggiore. Gravitational Waves. Vol. 1: Theory and Experiments. Oxford Master Series in Physics. Oxford University Press, 2007.

[105] Michele Maggiore. Gravitational Waves: Volume 1: Theory and Experiments, volume 1. Oxford university press, 2008.

[106] Michele Vallisneri. Use and abuse of the fisher information matrix in the assessment of gravitational-wave parameterestimation prospects. Physical Review D, 77(4):042001, 2008. 